\title{
Hair Texture Abnormal, CTCAE
}

National Cancer Institute

\section{Source}

National Cancer Institute. Hair Texture Abnormal, CT CAE. NCI Thesaurus. Code C143245.

A disorder characterized by a change in the way the hair feels. 